\title{
NS1619 regulates the expression of caveolin-1 protein in a time-dependent manner via ROS/PI3K/PKB/FoxO1 signaling pathway in Brain Tumor Microvascular Endothelial Cells
}

\author{
Rui-ping Cai ${ }^{1}$, Yi-xue Xue ${ }^{2}$, Jian Huang ${ }^{3}$, Jin-hui Wang $^{3}$, Jia-hong Wang ${ }^{1}$, Song-yan \\ Zhao $^{4}$, Ting-ting Guan ${ }^{1}$, Zhou Zhang ${ }^{1 *}$, Yan-ting Gu${ }^{1 *}$
}

${ }^{1}$ Department of Physiology, Life Science and Biopharmaceutical Institution, Shenyang Pharmaceutical University, Shenyang 110016, Liaoning Province, P.R. China

${ }^{2}$ Department of Neurobiology, College Basic of Medicine, China Medical University, Shenyang, 110001, Liaoning Province, P.R. China

${ }^{3}$ Department of Phytochemistry, Chinese Materia Medica Institution, Shenyang Pharmaceutical University, Shenyang 110016, Liaoning Province, P.R. China

${ }^{4}$ Department of Pharmacology experiment center, Life Science and Biopharmaceutical Institution, Shenyang Pharmaceutical University, Shenyang 110016, Liaoning Province, P.R. China

The final number of the text pages of the whole manuscript is 17 , and the number of figures is 4

*Corresponding Author: Zhou Zhang and Yan-ting Gu

E-mail: zzhouzhang@163.com (ZZ)

yanting-gu@163.com (Gu YT) 
NS1619 regulates the expression of caveolin-1 protein in a time-dependent manner via ROS/PI3K/PKB/FoxO1 signaling pathway in Brain Tumor Microvascular Endothelial Cells

Rui-ping Cai ${ }^{1}$, Yi-xue Xue ${ }^{2}$, Jian Huang ${ }^{3}$, Jin-hui Wang ${ }^{3}$, Jia-hong Wang ${ }^{1}$, Song-yan Zhao ${ }^{4}$, Ting-ting Guan ${ }^{1}$, Zhou Zhang ${ }^{1 *}$, Yan-ting Gu${ }^{1 *}$

\section{Abstract}

NS1619, a Calcium-activated potassium channel ( $K_{\mathrm{ca}}$ channel) activator, can selectively and time-dependently accelerate the formation of transport vesicles in both the brain tumor capillary endothelium and tumor cells within 15 min of treatment and then increase the permeability of the Blood-brain tumor barrier (BTB). However, the mechanism involved is still under investigation. Using a rat brain glioma (C6) model, the expression of caveolin-1, FoxO1 and p-FoxO1 protein were examined at different time points after intracarotid infusion of NS1619 at a dose of $30 \mu \mathrm{g} / \mathrm{kg} / \mathrm{min}$. Internalization of Cholera toxin subunit (CTB) labeled fluorescently was monitored by flow cytometry. The expression of caveolin-1 and FoxO1 protein at tumor microvessels was enhanced and caveolae-mediated CTB endocytosis was increased by NS1619 infusion for $15 \mathrm{~min}$. Compared with the $15 \mathrm{~min}$ group, the expression of caveolin-1 protein was significantly decreased and the level of phosphorylation of FoxO1 was significantly increased in the NS1619 2 hrs group. In addition, inhibitors of Reactive Oxygen Species (ROS) or PI3K or PKB significantly attenuated the level of FoxO1 phosphorylation and also increased the expression of caveolin-1 protein in Human Brain Microvascular Endothelial Cells (HBMECs) cocultured with human glioma cells (U87) 2 hrs after NS1619 treatment. This led to the conclusion that NS1619-mediated transport vesicle increase is, at least partly, related to the ROS/PI3K/PKB/FoxO1 signaling pathway.

Keywords: Blood-brain tumor barrier, NS1619, Glioma, Caveolin-1, FoxO1 


\section{Introduction}

Brain gliomas are one of the most common tumors of the Central Nervous System (CNS) and are second highest in causing mortality rate among brain tumors, which makes it an important area to be focused on [1,2]. The blood-brain barrier (BBB) mainly consists of microvascular endothelial cells and overlying astrocytic foot processes, which regulates the entry of blood-borne molecules into brain parenchyma, and preserves ionic homeostasis into the brain microenvironment [3]. However, the existence of BBB may impede the entry of antitumor drugs in the tumor tissue. Thus, selective opening of the BBB is crucial for the entry of anti-cancer drugs into the brain tumor tissue, which is a pre-requisite for chemotherapy of brain tumors [4]. Ningaraj et al. demonstrated that NS1619, a $K_{\mathrm{Ca}}$ channel activator, could selectively increase the blood-brain tumor barrier (BTB) permeability without affecting normal BBB [5]. However, the mechanisms responsible for this NS1619-induced effect are still unclear. Particularly, in light of a number of prior $\mathrm{BBB}$ disruption putative therapeutics have been employed without clinical effect in glioma therapy. Consequently, it would be important to understand the mechanism of NS1619 on glioma cerebrovasculature and might perspectively contribute to an interesting action of antitumor therapy.

Caveolae are the small plasma membrane invaginations, involved in cell transport, substance metabolism and signal transduction, and depends on the presence of a family of proteins called caveolins for its activity [6]. Caveolin-1 is the most abundant structural protein which is required for the caveolae formation, and is also very essential in regulating the activity and localization of signaling molecules involved in vesicle fission, endocytosis, fusion, and exocytosis [7,8]. Changes in the level of caveolin-1 expression is associated with the regulation of BBB permeability [9]. Also NS1619 accelerates the formation of transport vesicles in both the brain tumor capillary endothelium and tumor cells within 15 minutes of treatment [10] and after $2 \mathrm{~h}$, it begins to open the tight junction (TJ) [11]. However, the mechanism by which this occurs is still under investigation. FoxO1 is an important effect factor of phosphoinositide-3 kinase/protein kinase B (PI3K/PKB) signaling pathways and gene 
transcription regulation switch. The expression of target gene can be induced or inhibited by FoxO1 phosphorylation and dephosphorylation [12,13]. Van et al. confirmed that FoxO1 interaction with the caveolin-1 promoter sequence regulates the expression of caveolin-1[14]. It has been found that C-terminus of alpha subunit of human large-conductance calcium-activated potassium channels (hSlo1) are critical for hSlo1c/Cav-1 interaction [15]. Moreover, we have shown that NS1619 increases BTB permeability in a time-dependent manner by down-regulating TJ protein expression and this effect is related to ROS/RhoA/PI3K/PKB signaling pathway [11]. Based on above-mentioned results, we hypothesize that NS1619 could enhance the formation of transport vesicles of brain tumor microvessels through regulating the expression of caveolin-1 and this time-dependent effect could be related to the ROS/PI3K/PKB/FoxO1 signaling pathway. To test the idea, we utilized in vivo and in vitro BTB model and evaluated the expression and distribution of caveolin-1, FoxO1 and p-FoxO1 protein at different time points.

\section{Methods}

\section{Experiments in vivo}

\section{Animals implanted with rat brain glioma cells (C6 cells)}

Adult male Wistar rats (body weight 180-220g) bought from the Center for Experimental Animals of Shenyang Pharmaceutical University were used for the present study. All animal experiments were conducted as per the National Institute of Health Guide for the Care and Use of Laboratory Animals as well as the policies of our university authority. At first, the rats were anesthetized and then intracerebrally injected with $1 \times 10^{6}$ C6 cells in $10 \mu$ l of medium using a Hamilton syringe. The coordinates were $1 \mathrm{~mm}$ anterior bregma, $3 \mathrm{~mm}$ lateral to the midline and $5.5 \mathrm{~mm}$ deep to the caudate nucleus. Ten days after tumor implantation, the rats were prepared for the following experiments.

\section{Treatment with NS1619}

Through a ventral neck midline incision, the common and external carotid arteries ipsilateral to the tumor were isolated. Both the distal end of the external carotid artery and the proximal end of the common carotid artery were ligated. NS1619 
(Sigma-Aldrich) was pumped into the brain via the proximal end of the common carotid artery at a dosage of $30 \mu \mathrm{g} / \mathrm{kg} / \mathrm{min}$ as per the study carried out by Ningaraj et al.[5]. Six time point groups were selected for Western blot investigation after NS1619 infusion from $0 \mathrm{~min}, 15 \mathrm{~min}, 30 \mathrm{~min}, 1 \mathrm{hr}, 2 \mathrm{hr}$ and $4 \mathrm{hr}$. Six rats from each group were used in the following experiments.

\section{Immunohistochemistry}

The rats of the NS1619 0 min group, NS1619 15 min group and NS1619 2 hr group were deeply anesthetized with chloral hydrate and transcardially perfused with saline and $4 \%$ paraformaldehyde, then the tissues were placed in $20 \%$ and $30 \%$ sucrose, respectively. The sections were stained with hematoxylin and eosin to confirm the presence of tumor. The sections were immunohistochemically stained with the mouse monoclonal antibody anti-caveolin-1 (diluted 1:200; BD Biosciences) following standard procedures. For semi-quantitative measurements of caveolin-1 protein density, the slides were photographed (IX71,Olympus, Japan) and measured using a computer-aided image analyzing system (Motic Images Advanced 3.2).

\section{Western blot analysis}

The effect of NS1619 on caveolin-1, FoxO1 and p-FoxO1 protein was analyzed through Western blotting. Brain tumor tissue of rats from different time point groups were removed and tumor microvessels were extracted. Protein homogenates of tumor microvessel samples were prepared by rapid homogenization in lysis buffer with PMSF (Beyotime Biotechnology, Beijing, China). Samples were centrifuged at 12000 $\mathrm{g}$ for $20 \mathrm{~min}\left(4^{\circ} \mathrm{C}\right)$. The Protein lysates were fractioned on $10 \%$ NuPAGE Bis-Tris gels. iBlot Gel Transfer Device was used for dry blotting of protein from mini gels with iBlot Gel Transfer Stacks for 7-9 min according to molecular weight of protein. The membranes were incubated in blocking buffer (5\% non-fat dairy milk dissolved in Tween-Tris-buffered saline, TTBS) for $2 \mathrm{hr}$ at room temperature. The blots were then incubated with rabbit polyclonal antibody anti-FoxO1 (dilution 1:1000, Thermo), rabbit polyclonal antibody anti-p-FoxO1 (dilution 1:800, Abcam), mouse monoclonal antibody $\beta$-actin (dilution 1:20000, Proteintech) and mouse monoclonal anti-caveolin-1 (dilution 1:800, BD Biosciences) overnight at $4^{\circ} \mathrm{C}$. The caveolin-1, 
FoxO1 and p-FoxO1 protein bands and $\beta$-actin on these immunoblots were visualized using enhanced chemiluminescence (ECL kit, Beyotime Biotechnology). $\beta$-actin was used as a control in the procedure. The protein bands were scanned using GIS ID Ver4.2 software and normalized to $\beta$-actin.

\section{Experiments in vitro}

\section{Establishment of BTB model in vitro}

Immortalized human cerebral microvascular endothelial cell line (HCMEC/D3) was a gift from Dr. Pierre-Olivier Couraud, Department of Neurology and Stroke Centre, Bichat University Hospital, Paris, France. Cells were grown in an EBM-2 medium (Lonza) containing 10\% Fetal Bovine Serum (gibico, Life technology),

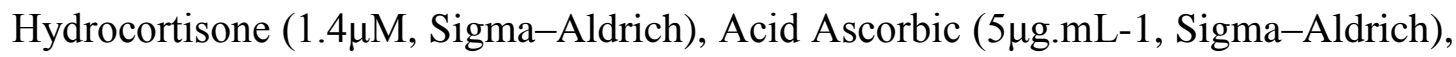
Chemically Defined Lipid Concentrate (1/100, Life technology), HEPES (10mM, Life technology), bFGF (1ng.mL-1, Sigma-Aldrich).

The BTB model was established in vitro as described by Hurst et al.[16] Initially, human U87 MG cells were plated on the inner-side of collagen-coated polytetrafluoroethylene polyester polystyrene transwell membrane (Corning, costar) with suitable culture medium containing $10^{6}$ cells. When U87 MG cells were $80 \%$ confluent, HCMEC/D3 were plated on the upper side of the transwell membrane. The cells were then allowed to achieve $80 \%$ confluency and co-cultured for 5 days.

\section{Drug treatment}

In order to attain the specific inhibition of the activity of ROS/PI3K/PKB, MPG, ROS inhibitor $(10 \mu \mathrm{M}$, Sigma-Aldrich) or LY294002, PI3K inhibitor $(2 \mu \mathrm{M}$, Sigma-Aldrich) or triciribine, PKB inhibitor (12.5 $\mu \mathrm{M}$, Sigma-Aldrich) was added to the upper compartment of the Transwell for 20 min. NS1619 (10 $\mu \mathrm{M}$, Sigma-Aldrich) was then added to upper compartment of the Transwell after the complete removal of inhibitor. The cultured cells were then rinsed with cold phosphate-buffered saline (PBS) at 15min or $2 \mathrm{~h}$ after NS1619 treatment. The HCMEC/D3 was used in the following experiments.

\section{Internalization of Cholera toxin subunit B (CTB)}


The method was established according to the previous study described by Erika et al. [17]. The medium of cells with treatment was removed and replaced by DMEM supplemented with $1 \%$ of serum-free N1 medium for $1 \mathrm{~h}$. CTB-FITC was added at 1 $\mu \mathrm{g} / \mathrm{mL}$ (Invitrogen, Life technology) for $1 \mathrm{~h}$ to enable determination of the fraction of labeled molecules or particles that was effectively internalized within cells. Cells were then washed in PBS and re-suspended in PBS containing 2\% formaldehyde. Internalization of fluorescently labeled CTB was monitored by flow cytometry.

\section{Western blot analysis}

The effect of specific inhibitor and NS1619 on p-FoxO1 and caveolin-1 protein expression in HCMEC/D3 was analyzed via Western blotting. The process has been introduced in the methods of in vivo experiment .

\section{Immunofluorescence assays}

The HCMEC/D3 monolayers, grown on 24-well plate were permeabilized with $0.2 \%$ Triton X-100 in PBS on ice for two minutes. Then fixed with 3\% paraformaldehyde for 30 minutes and permeabilized with $0.05 \%$ Triton X-100 for 5 minutes. After blocking with $5 \%$ bovine serum albumin in PBS for 20 mins, cells were incubated with primary antibodies against caveolin-1 protein to assess the expression of caveolin-1 protein. The cells were incubated with rhodamine-labeled or FITC-donkey anti-rabbit for $1 \mathrm{hr}$ at room temperature (diluted 1:1000, Invitrogen, Life technology). The images were then analyzed using Laser scanning confocal microscope (C2si, Japan).

\section{Statistical analysis}

All data are presented as the mean \pm SD. One-way analysis of variance (ANOVA) was used to compare the group differences in the measurements of protein expression. Dunnett's post hoc tests was used to compare specific group differences if the ANOVA revealed a significant difference. For other measurements, the data were assessed using paired Student's t test. $P<0.05$ was considered significant statistically.

\section{Results}

Impact of NS1619 on the intracellular accumulation of CTB in Human Brain 


\section{Microvascular Endothelial Cells with cocultured with glioma cells (GECs)}

Cholera toxin subunit B (CTB) is usually internalized through caveolae dependent endocytosis. Thus CTB-FITC is used as marker of caveolae-mediated endocytosis $[18,19]$. Flow cytometry measurement of intracellular fluorescence was done after $1 \mathrm{~h}$ exposure of glioma endothelial cells (GECs) with NS1619 treatment to $1 \mu \mathrm{g} / \mathrm{mL}$ CTB-FITC. Cellular uptake of CTB-FITC was significantly amplified in NS1619 15 min group (Figure 1). These data thus established for the first time that NS1619 acts as a modulator of caveolae-mediated endocytosis.

\section{NS1619 time-dependently induced the over-expression of caveolin-1 protein in rat}

\section{C6 brain glioma model}

NS1619 infusion in rat C6 brain tumor tissues at $0 \mathrm{~min}$, induced lower or weak expression of caveolin-1 protein. The expression of caveolin-1 protein was greatly increased at $15 \mathrm{~min}$ after infusion. The level of caveolin-1 protein was also significantly reduced at $2 \mathrm{hr}$ after NS1619 infusion and began to increase again at $4 \mathrm{hr}$ after treatment (Figure 2A and 2B). The localization of caveolin-1 protein was observed by Immunohistochemistry. Caveolin-1 was localized at brain tumor capillary endothelium and tumor cells. In NS1619 0 min group, the expression of caveolin-1 is lower or weak, and found to be increased in brain tumor capillary endothelium at 15 min after NS1619 infusion. At $2 \mathrm{hr}$ after NS1619 administration, the immunoreactivity of caveolin-1 was greatly attenuated ((Figures 2G1-G3. G1-1, G2-1 and G3-1 are enlarged areas for better visualization). Quantification of caveolin-1 immunoreactivity is presented on Figure 2H.

\section{NS1619 time-dependently induced the expression of FoxO1 and p-FoxO1 proteins in rat $\mathrm{C6}$ brain glioma model}

In rat $\mathrm{C} 6$ brain tumor tissues, the expression of FoxO1 protein was abundant in $0 \mathrm{~min}$ group and significantly increased at 15 min after infusion and lasted until $4 \mathrm{hr}$ after NS1619 treatment (Figure 2A and C). In addition, p-FoxO1 was slightly expressed in NS1619 0 min group. However, the expression of p-FoxO1 protein began to increase 
significantly at $1 \mathrm{hr}$ and was at peak $2 \mathrm{hr}$ after NS1619 infusion. Subsequently, the level of $\mathrm{p}$-FoxO1 protein began to decrease at $4 \mathrm{~h}$ after infusion but the decrease was not statistically significant. In addition, we demonstrated that the ROS inhibitor, MPG greatly reduces the up-regulation of p-FoxO1 protein at $1 \mathrm{hr}, 2 \mathrm{hr}$ and $4 \mathrm{hr}$ after NS1619 infusion (Figure 2E and F).

The ROS/PI3K/PKB pathway is involved in the NS1619-induced changes in caveolin-1 expression in vitro

The effect of specific inhibitors and NS1619 on the expression of caveolin-1 protein in HCMEC/D3 in vitro BTB model was analyzed via Western blotting. MPG or LY294002 or triciribine did not prevent the up-regulation of caveolin-1 in GECs for $15 \mathrm{~min}$, but reversed the down-regulation of caveolin-1 at $2 \mathrm{hr}$ after NS1619 infusion (Figure 3A, D and G). The IDV ratios of caveolin-1 for western blots in different groups were shown in (Figure 3B, E and H).

The localization of caveolin-1 was observed by Immunofluorescence microscopy. Caveolin-1 was mainly localized to the cell cytoplasm. In NS1619 0 min group, the expression of caveolin-1 is lower or weak, and found to be increased in the nucleus at 15 min after NS1619 infusion. At 2 hr after NS1619 administration, the immunoreactivity of caveolin-1 was greatly attenuated. In addition, MPG, LY294002 or triciribine partially prevented the decrease of caveolin-1 induced by NS1619 treatment for $2 \mathrm{hr}$ (Figure 4).

The ROS/PI3K/PKB pathway is involved in the NS1619-induced FoxO1 phosphorylation

We previously studied the activation of signaling cascades involving ROS/PI3K/PKB in BTB permeability increase induced by NS1619. To investigate the effect of ROS/PI3K/PKB signaling pathway on the level of FoxO1 phosphorylation in GECs, we evaluated the effect of MPG or LY294002 or triciribine on the phosphorylation state of FoxO1 by Western blotting. Pretreatment with MPG or triciribine or LY294002 significantly inhibited the phosphorylation of FoxO1 at $2 \mathrm{hr}$ after NS1619 treatment, but did not change the level of p-FoxO1 in GECs induced by NS1619 
treatment for $15 \mathrm{~min}$. The IDV ratios of FoxO1 phosphorylation for western blots were shown in Figure $3 \mathrm{C}, \mathrm{F}$ and I.

\section{Discussion}

The use of NS1619 as a Kca channel activator in rat glioma (C6) model has been shown to accelerate selectively and time-dependently the formation of transport vesicles in both the brain tumor capillary endothelium and tumor cells and increase the permeability of BTB $[5,20]$. In order to further understand the related mechanism, we examined the expression of caveolin-1, FoxO1 and p-FoxO1 protein from 0 min to 4 hours post NS1619 infusion. The results demonstrated that the NS1619 significantly increased the expression of caveolin-1 in a rat brain glioma (C6) model at 15 minutes and lasted until $1 \mathrm{hr}$ post infusion, accompanied with an increase of FoxO1 protein. Also the expression of p-FoxO1 was significantly increased and the expression of caveolin-1 protein was markedly decreased at $2 \mathrm{hr}$ after NS1619 treatment, which was partly prevented by specific inhibitors for ROS, PI3 kinase and PKB. This study for the first time demonstrated that that the mechanism of an increase in the formation of transport vesicles in a time-dependent manner induced by NS1619 could be related to the ROS/PI3K/PKB/FoxO1A/caveolin-1 signaling pathway.

Caveolin-1 is the most prominent marker of caveolae in brain vascular endothelial cells. It plays a key role in physiological and pathological conditions of the BBB and serves as a mediator in drug delivery through the BBB [21]. Caveolin-1 is related to the diminished expression of tight junction-associated proteins and metabolic pinocytosis vesicles when the BBB is disrupted by drugs or malignant stimulus [22-24]. These studies imply the prime role of caveolin-1 in the BBB and its profound implications for central nervous system disease and drug delivery through the BBB [25-28]. In rat C6 brain tumor model, we demonstrated that the expression of caveolin-1 protein will be at its peak at 15 min after NS1619 infusion compared with the 0 min group. Then the level of caveolin-1 protein significantly reduces at $2 \mathrm{hr}$ after infusion and starts to reverse at $4 \mathrm{hr}$. In addition, we demonstrated that cellular uptake of CTB-FITC was significantly amplified in NS1619 15 min group, suggesting that NS1619 acts as a modulator of caveolae-mediated endocytosis. Thus, it can be 
depicted that NS1619 might regulate the expression of caveolin-1 protein in a time-dependent manner. However, the mechanism should be further investigated. FoxO (Forkhead Box O) is a subfamily of Fox gene transcription factors. FoxO1 is an important regulatory factor involved in the functioning of vascular endothelial cells [12]. The study demonstrated that when FoxO1 combines with the promoter sequence of the caveolin-1 gene, directly regulates the expression of caveolin-1[14]. Our present study confirmed that NS1619 significantly up-regulates the expression FoxO1 and caveolin-1 protein at 15 min after infusion, which further indicates that FoxO1 can be involved in BTB permeability by promoting the transcriptional regulation of caveolin-1. It is hard to imagine that the overall caveolin-1 signal increases dramatically at 15 min after NS1619 infusion on the brain sections only by protein translation. We speculate NS1619 could also inhibit the degradation of caveolin-1 protein via downregulating the special miRNA targeted to 3'UTR of caveolin-1. In addition, we also demonstrated that there is no significant difference between the expression of FoxO1 protein at 15 minutes and $2 \mathrm{hr}$ post infusion. However, it is interesting that the expression of caveolin-1 was significantly increased at 15 minutes, and then markedly decreased at $2 \mathrm{hr}$ after NS1619 treatment. The mechanism should be furtherly investigated.

FoxO1 transcription factors are usually located at the nucleus, where they can bind to the promoter regions of target genes and regulate their protein expression. After phosphorylation FoxO1 migrates to the cytoplasm and becomes transcriptionally inactive. Fox $\mathrm{O} 1$ is an important effector of phosphoinositide-3 kinase/protein kinase $\mathrm{B}(\mathrm{PI} 3 \mathrm{~K} / \mathrm{PKB})$ signaling pathways and gene transcription regulation switch. The expression of target genes can be induced or inhibited by FoxO1 phosphorylation and dephosphorylation. We have shown before that NS1619 could time-dependently activate the ROS/PI3K/PKB signaling pathway. Thus, we hypothesized that the activation of ROS/PI3K/PKB signaling pathway could lead to an increase in level of FoxO1 phosphorylation, and then FoxO1 would be transferred to the cytoplasm with transcriptional inactivity. Therefore, we examined the expression of p-FoxO1 protein at different time points after NS1619 infusion, and found that the phosphorylation 
level of FoxO1 began to increase at $1 \mathrm{~h}$ and reached a maximum at 2 hours. In addition, it is demonstrated that reactive oxygen species can enhance the effects of tissue type plasminogen activator on MMP activation through the loss of caveolin-1 that serves as a critical determinant of BBB permeability [29]. Our previous studies have shown that ROS/PI3K/PKB signaling pathway was activated at $1 \mathrm{~h}$ after NS1619 treatment [11]. Therefore, we utilized in vitro BTB model and investigated the effect of specific inhibitor and NS1619 on p-FoxO1 and caveolin-1 protein expression. The results demonstrated that the inhibitor of ROS or PI3K or PKB significantly attenuated the expression of p-FoxO1 and increased the expression of caveolin-1 protein in HCMECs/D3 cocultured with U87 after 2 hours of NS1619 treatment, suggesting that NS1619 might regulate the expression of caveolin-1 protein in a time-dependent manner and this effect could be related to the ROS/PI3K/PKB/FoxO1 signaling pathway.

In conclusion, the mechanism by which NS1619 induces BTB permeability changes is complex. Our results provide evidence that NS1619 regulates the expression of caveolin-1 protein in a time-dependent manner through the ROS/PI3K/PKB/FoxO1 signaling pathway, and provides a new target for understanding the operative mechanisms of NS1619-regulated BTB permeability and the delivery of molecules across brain tumor microvessels following biochemical modulation. We believe that NS1619 could potentially modulate the drug delivery in brain tumor therapy.

\section{Acknowledgments}

This work was supported by National Science Fundation for China, No.81201989, Special and scientific research foundation of Shenyang Pharmaceutical University for Middle and young teachers' career development, No.ZCJJ2014407; National Science Fundation for Liaoning Province, No. 2015020564. Innovative entrepreneurship training plan of Shenyang Pharmaceutical University.

\section{Disclosure}

The authors confirm that there are no disclosures, no conflicts of interest and no financial supports to declare. 


\section{References}

1. Drappatz J., Schiff D., Kesari S., Norden A.D., Wen P.Y. (2007) Medical management of brain tumor patients. Neurol Clin. 25: 1035-1071.

2. Hayashi Y., Yoshida Y., Hamada J.(2006) Blood-tumor barrier in malignant brain tumor. No Shinkei Geka. 34:983-999.

3. Svetlana, M., Stamatovic, Richard F. Keep, Anuska, V., Andjelkovic (2008) Brain Endothelial Cell-Cell Junctions: How to "Open” the Blood Brain Barrier.Current Neuropharmacology. 6: 179-192.

4. Komarova, Y., Malik, A.B. (2010) Regulation of endothelial permeability via paracellular and transcellular transport pathways. Annu Rev Physiol. 72:463-93.

5. Ningaraj N.S., Rao M., Hashizume K., Asotra K., Black K.L. (2002) Regulation of blood-brain tumor barrier permeability by calcium activated potassium channels. J Pharmacol Exp Ther. 301:838-851.

6. Quest A.F., Gutierrez-Pajares J.L., Torres V.A. (2008) Caveolin-1: an ambiguous partner in cell signalling and cancer. J Cell Mol Med. 12: 1130-1150.

7. Sun S.W., Zu X.Y., Tuo Q.H., Chen L.X., Lei X.Y., Li K., Tang C.K., Liao D.F.(2010) Caveolae and caveolin-1 mediate endocytosis and transcytosis of oxidized low density lipoprotein in endothelial cells. Acta Pharmacol Sin. 31:1336-42.

8. Drab M, Verkade P, Elger M, Kasper M, Lohn M, Lauterbach B, Menne J, Lindschau C, Mende F, Luft FC, Schedl A, Haller H, Kurzchalia TV (2001) Loss of caveolae, vascular dysfunction, and pulmonary defects in caveolin-1 gene-disrupted mice. Science. 293(5539): 2449-2452.

9. Gu, Y., Dee, C., and Shen, J. (2011) Interaction of free radicals, matrix metalloproteinases and caveolin-1 impacts blood-brain barrier permeability. Front. Biosci. 3:1216-1231.

10. Ningaraj NS, Sankpal UT, Khaitan D, Meister EA, Vats TS (2009) Modulation of $\mathrm{KCa}$ channels increases anticancer drug delivery to brain tumors and prolongs survival in xenograft model. Cancer Biol Ther. 8(20):1924-33.

11. Gu YT, Xue YX, Wang YF, Wang JH, ShangGuan QR, Zhang JX, Qin LJ (2012) 
Role of ROS/RhoA/PI3K/PKB signaling in NS1619-mediated blood-tumor barrier permeability increase. J Mol Neurosci. 48(1):302-12.

12. Ferdous A., Morris J., Abedin M.J., Collins S., Richardson J.A., Hill J.A. (2011) Forkhead factor FoxO1 is essential for placental morphogenesis in the developing embryo. Proc Natl Acad Sci U S A. 108(39):16307-12

13. Herzog S., Hug E., Meixlsperger S., Paik J.H., DePinho R.A., Reth M., Jumaa H., (2008) SLP-65 regulates immunoglobulin light chain gene recombination through the PI(3)K-PKB-Foxo pathway. Nat Immunol. 9(6):623-31.

14. van den Heuvel A.P., Schulze A., Burgering B.M.(2005) Direct control of caveolin-1 expression by FOXO transcription factors. J Biochem. 385:795-802.

15. Song Y., Wang P., Ma J., Xue Y.(2014) C-terminus of human BKca channel alpha subunit enhances the permeability of the brain endothelial cells by interacting with caveolin-1 and triggering caveolin-1 intracellular trafficking. Neuromolecular Med. 16(2):499-509.

16. Hurst, R.D. and Fritz, I.B., (1996) Properties of an immortalised vascular endothelial/glioma cell co-culture model of the blood-brain barrier. J Cell Physiol. 167(1), 81-8.

17. Bourseau-Guilmain E, Griveau A, Benoit JP, Garcion E. (2011) The importance of the stem cell marker prominin-1/CD133 in the uptake of transferrin and in iron metabolism in human colon cancer Caco-2 cells. PLoS One. 6(9):e25515.

18. Orlandi PA, Fishman PH (1998) Filipin-dependent inhibition of cholera toxin: evidence for toxin internalization and activation through caveolae-like domains. $\mathbf{J}$ Cell Biol 141: 905-915.

19. Conner SD, Schmid SL (2003) Regulated portals of entry into the cell. Nature 422: 37-44.

20. Gu Y.T., Xue Y.X., Wang Y.F., Wang J.H., Chen X., ShangGuan Q.R., Lian Y., Zhong L., Meng Y.N.(2013) Minoxidil sulfate induced the increase in blood-brain tumor barrier permeability through ROS/RhoA/PI3K/PKB signaling pathway. Neuropharmacology. 75:407-15.

21. Kim, M., Park, S., Kopetz, S., and Gallick, G., (2009) Src family kinases as 
mediators of endothelial permeability: effects on inflammation and metastasis. Cell Tissue Res. 335: 249-259.

22. Liu, L., Xue, Y., and Liu, Y (2010) Bradykinin increases the permeability of the blood-tumor barrier by the caveolaemediated transcellular pathway. J. Neuro-oncol. 99: 187-194.

23. Liu, J., Jin, X., Liu, K.J., and Liu, W. (2012) Matrix metalloproteinase-2 mediated occludin degradation and caveolin-1-mediated claudin-5 redistribution contribute to blood-brain barrier damage in early ischemic stroke stage. J. Neurosci. 32:3044-3057.

24. Gu, Y., Zheng, G., Xu, M., Li, Y., Chen, X., Zhu, W., Tong, Y., Chung, S.K., Liu, K.J., and Shen, J. (2012) Caveolin-1 regulates nitric oxide-mediated matrix metalloproteinases activity and blood-brain barrier permeability in focal cerebral ischemia and reperfusion injury. J. Neurochem. 1: 147-156.

25. Zhao Y.L., Song J.N., Zhang M., 2014. Role of caveolin-1 in the biology of the blood-brain barrier. Rev Neurosci. 25(2): 247-54.

26. Madaro L., Antonangeli F., Favia A., Esposito B., Biamonte F., Bouché M., Ziparo E., Sica G., Filippini A., D'Alessio A.(2013) Knock down of caveolin-1 affects morphological and functional hallmarks of human endothelial cells. J Cell Biochem. 114(8):1843-51.

27. Minshall R.D., Sessa W.C., Stan R.V. (2003) Caveolin regulation of endothelial function. Am J Physiol Lung Cell Mol Physiol. 285(6):L1179-1183.

28. Wang, P., Xue, Y., Shang, X., and Liu, Y. (2010) Diphtheria toxin mutant CRM197-mediated transcytosis across blood-brain barrier in vitro. Cell. Mol. Neurobiol. 30:717-725.

29. Lakhan, S.E., Kirchgessner, A., Tepper, D., and Leonard, A. (2013) Matrix metalloproteinases and blood-brain barrier disruption in acute ischemic stroke. Front. Neurol. 4:32.

Fig.1. NS1619 induced an increase in Cholera toxin subunit B (CTB) intracellular accumulation in the glioma endothelial cells (GECs). Flow cytometric analysis of intracellular uptake of CTB-FITC in NS1619 0 min, 15 
min and $2 \mathrm{hr}$. Results are expressed as percentage of control, thus representing the geomean fluorescence intensity levels obtained for cells treated with vehicle alone.

Fig.2. Effects of NS1619 on caveolin-1, FoxO1A and p-FoxO1A (phospho S256) protein expression in the rat brain tissue at different time point after NS1619 infusion (A). Lane 1: NS1619 0 min group; lane 2: NS1619 15 min group; Lane 3: NS1619 30 min group; Lane 4: NS1619 1 hr group; Lane 5: NS1619 2 hr group; Lane 6: NS1619 4 hr group. Representative Western blots illustrating differences in the bands of caveolin-1, FoxO1A and p-FoxO1 protein. Changes of relative integrated density value (IDV) of caveolin-1 (B), FoxO1A (C) and p-FoxO1 (D) protein ( $n=6$, each). ${ }^{*} P<0.05,{ }^{* *} P<0.01$ vs. NS1619 0 min group, ${ }^{\# \#} P<0.01$ vs. NS1619 15 min group. Effects of MPG on NS1619-induced increasing protein levels of p-FoxO1 in the brain tumor tissues of the rat (E). Lane 1: NS1619 $1 \mathrm{hr}$ group; lane 2: MPG+NS1619 $1 \mathrm{hr}$ group; Lane 3: NS1619 2 hr group; Lane 4: MPG+NS1619 2 hr group; Lane 5: NS1619 4 hr group; Lane 6: MPG+NS1619 4 hr group. IDV of p-FoxO1 (F) are shown. Data are presented as mean \pm S.D. $\left(n=6\right.$, each). ${ }^{*} P<0.01$ vs. NS1619 1 h group; ${ }^{\# \#} P<0.01$ vs. NS1619 2 h group; ${ }^{*} P<0.01$ vs. NS1619 4 h group. (G)Representative images of caveolin-1 immunohistochemistry. (G1) NS1619 0 min group; (G2) NS1619 15 min group; (G3) NS1619 2 hr group. G1-1, G2-1, G3-1 are enlarged images. Quantification of $\mathrm{G}$ is shown on $\mathrm{H}(n=5$, each).Scale bar: $20 \mu \mathrm{m}(\mathrm{H}){ }^{*} P<0.05$ vs. NS1619 0 min group, ${ }^{\#} P<0.05$ vs. NS1619 15 min group.

Fig.3. Effects of NS1619-induced change of protein levels of caveolin-1 and p-FoxO1 in glioma endothelial cells (GECs). (A)Lane 1: NS1619 0 min group; lane 2: NS1619 15 min group; Lane 3: NS1619 2 hr group;The integrated density values (IDV) of caveolin-1 (B) and p-FoxO1 (C) are shown. Data are presented as mean \pm S.D. ( $n=6$, each). ${ }^{* *} P<0.01$ vs. NS1619 0 min group, 
${ }^{\# \#} P<0.01$ vs. NS1619 15 min group. Effects of specific inhibitors on NS1619-induced change of protein levels of caveolin-1 and p-FoxO1 in GECs (D) .Lane 1: NS1619 15 min group; lane 2: MPG+NS1619 15 min group; Lane 3: LY294002+NS1619 15 min group; Lane 4: triciribine+NS1619 15 min;The integrated density values (IDV) of caveolin-1 (E) and p-FoxO1 (F) are shown. Data are presented as mean \pm S.D. ( $n=6$, each). Effects of specific inhibitors on NS1619-induced change of protein levels of caveolin-1 and p-FoxO1 in GECs .(G) Lane 1: NS1619 2 hr group; lane 2: MPG+NS1619 2 hr group; Lane 3: LY294002+NS1619 $2 \mathrm{hr}$ group; Lane 4: triciribine+NS1619 $2 \mathrm{hr}$. The integrated density values (IDV) of caveolin-1 (H) and p-FoxO1 (I) are shown. Data are presented as mean \pm S.D. ( $n=6$, each). ${ }^{*} P<0.05,{ }^{* *} P<0.01$ vs.NS1619 $2 \mathrm{~h}$ group.

Fig.4. Immunofluoresence localization of caveolin-1 protein in human brain microvascular endothelial cells (HBMECs) co-cultured with U87 (GECs) after NS1619 administration with/without specific inhibitors pretreatment. A: NS1619 0 min group; B: NS1619 15 min group; C: NS1619 2 hr group; D: LY294002+NS1619 2 hr group; E: triciribine+NS1619 2 hr group; The reduction of caveolin-1 protein induced by NS1619 treatment for $2 \mathrm{hr}$ was partially inhibited by specific inhibitor pretreatment. Scale bar: $20 \mu \mathrm{m}$. 


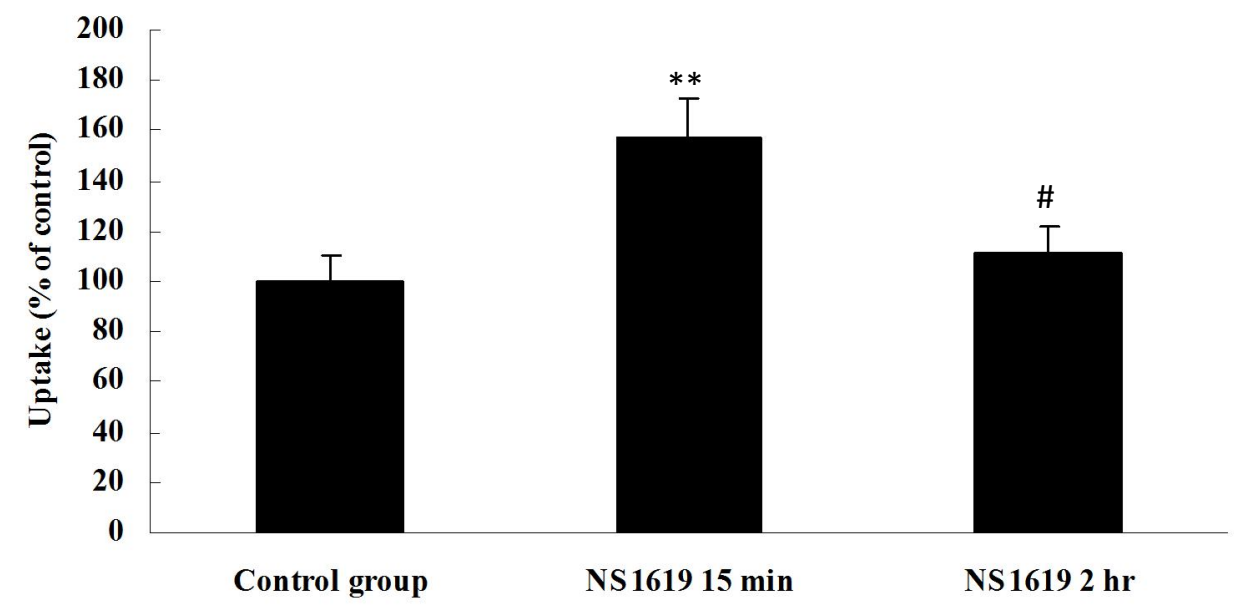

Cholera toxin B Subunit-FITC 

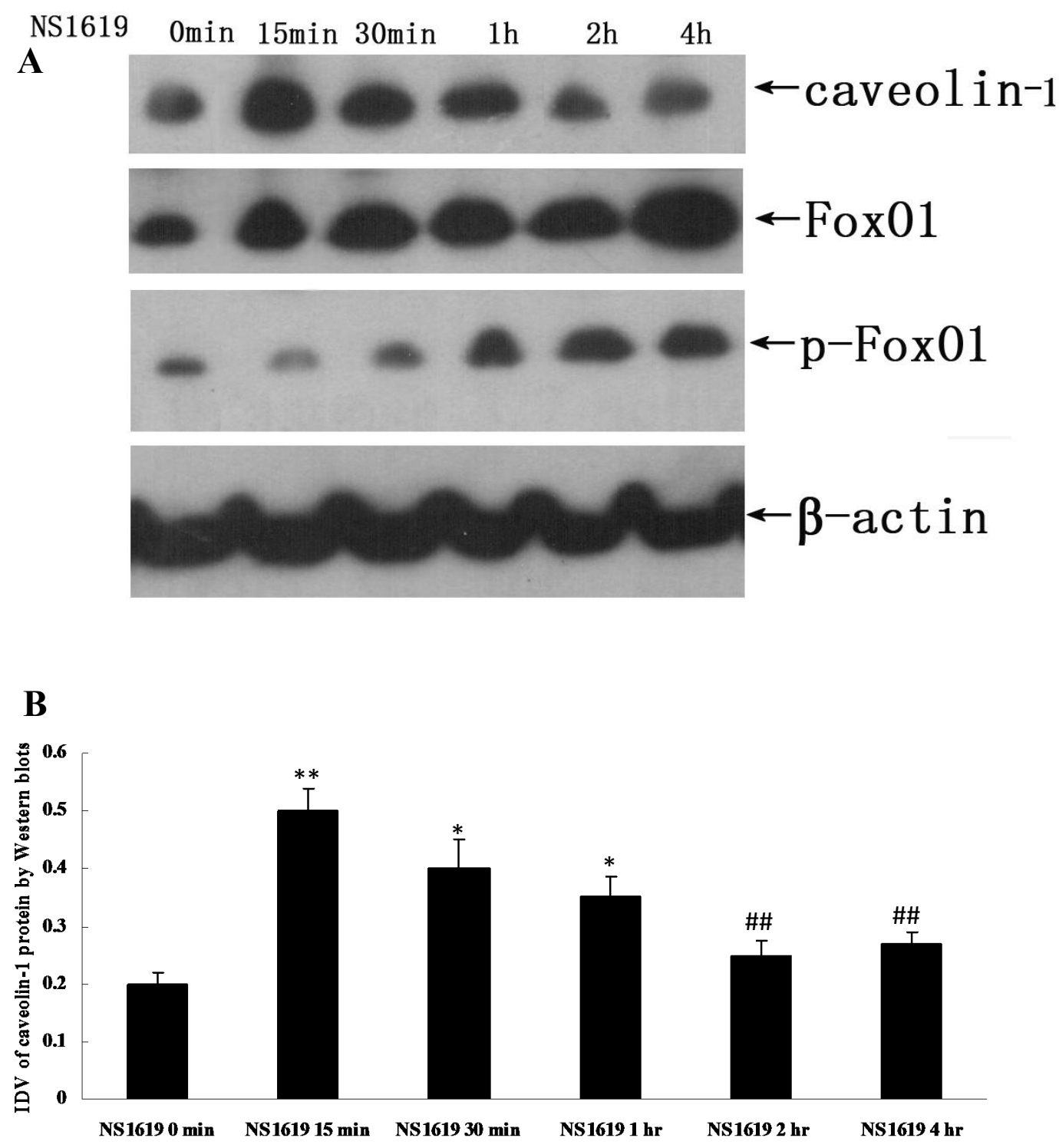

\section{C}

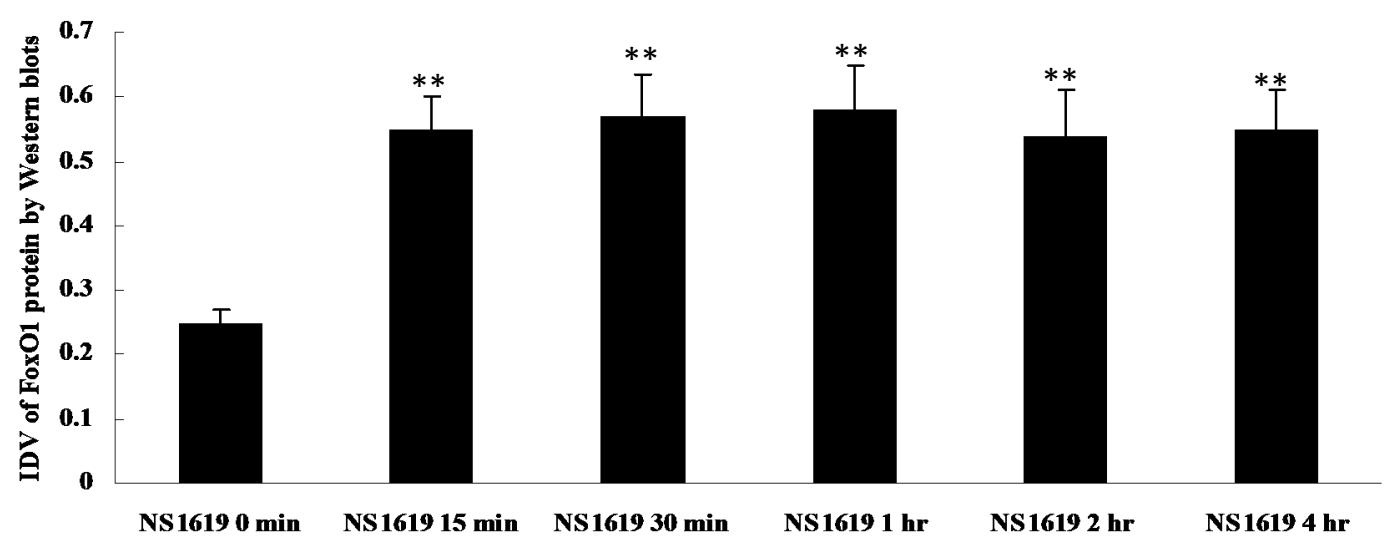


D

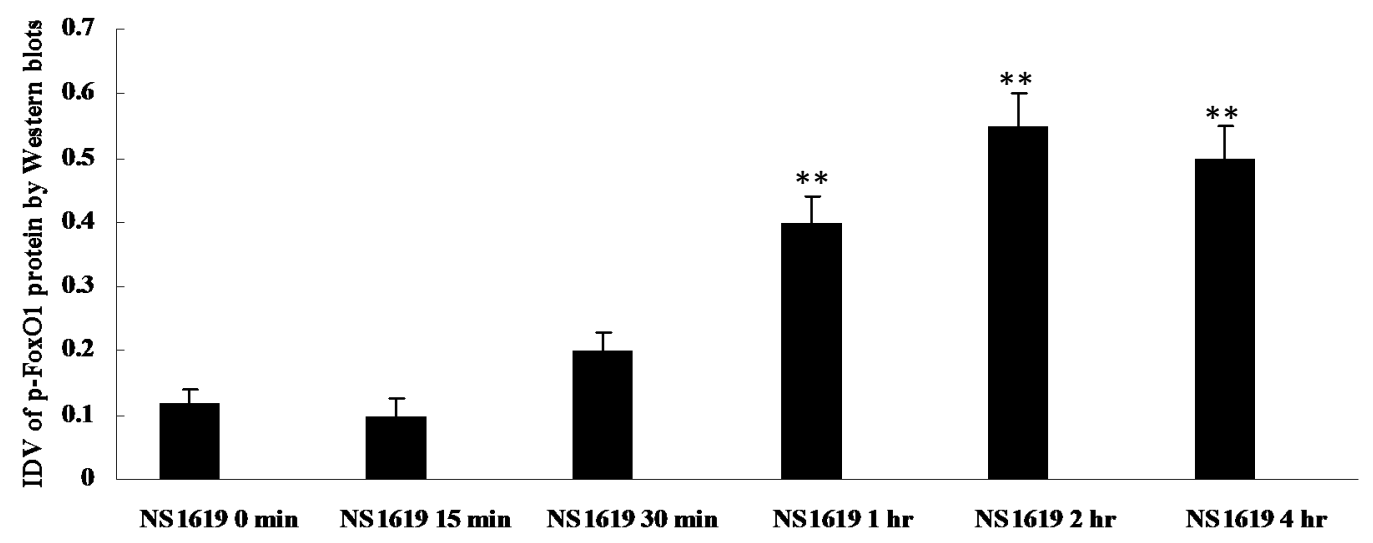

E

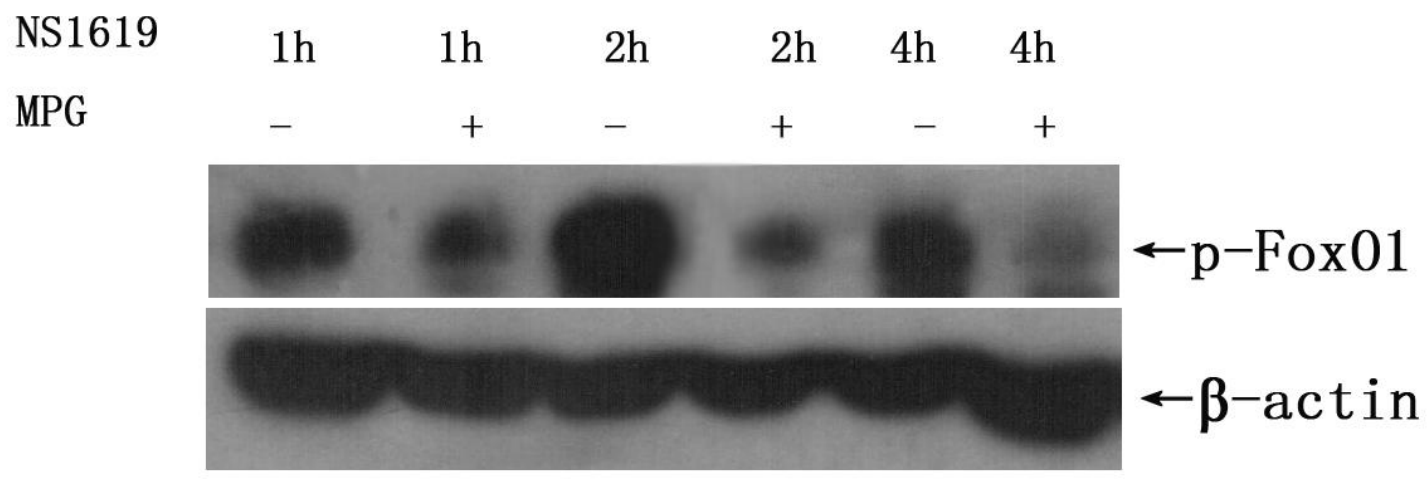

F

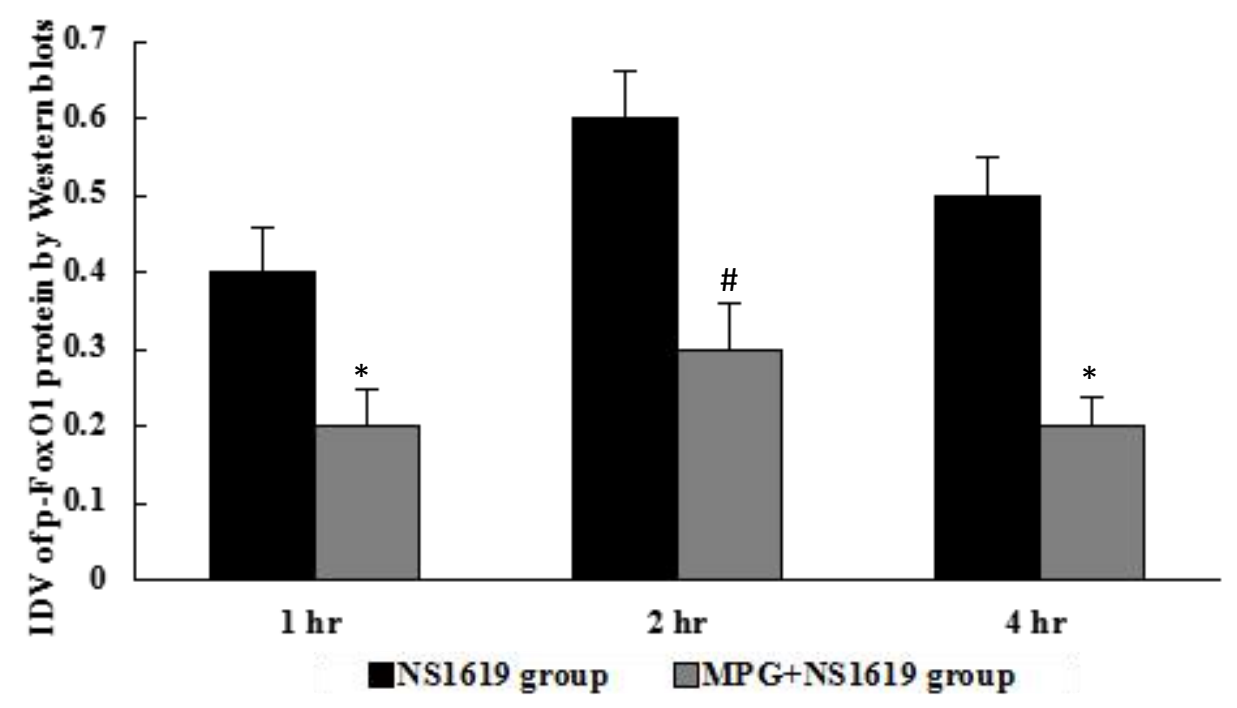



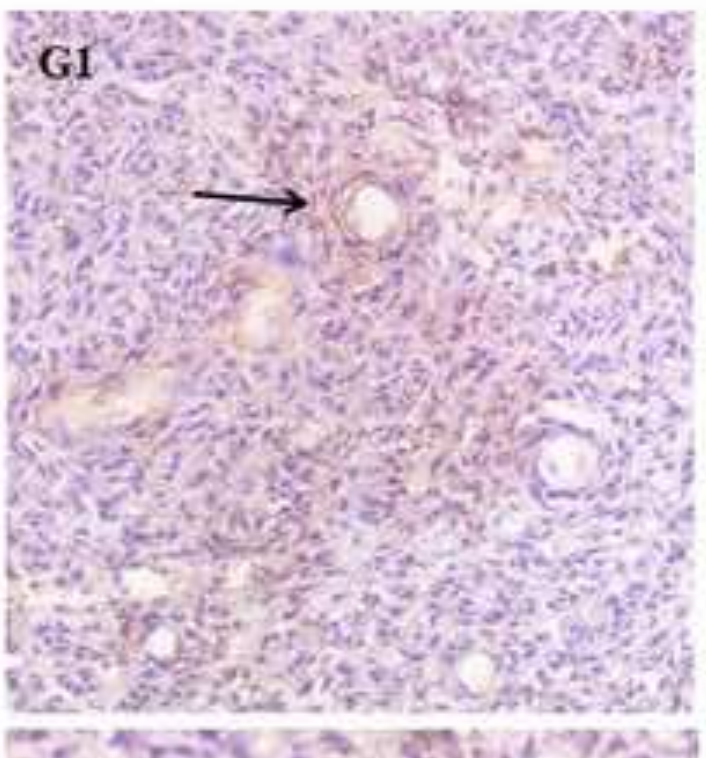

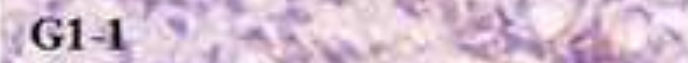

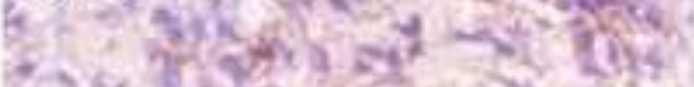

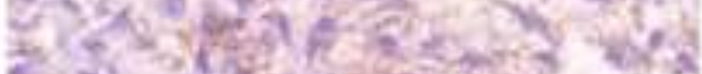

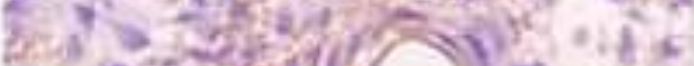

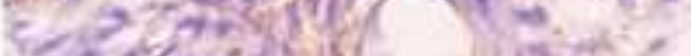

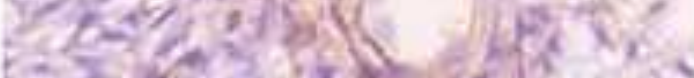

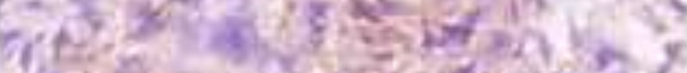

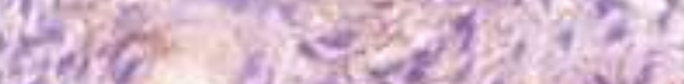

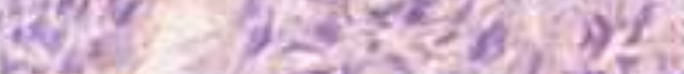

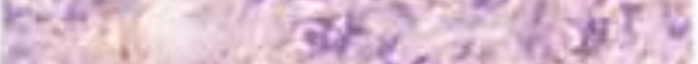

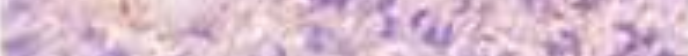

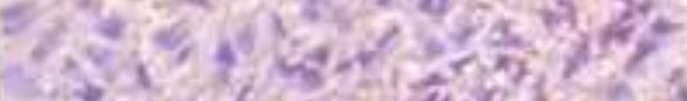

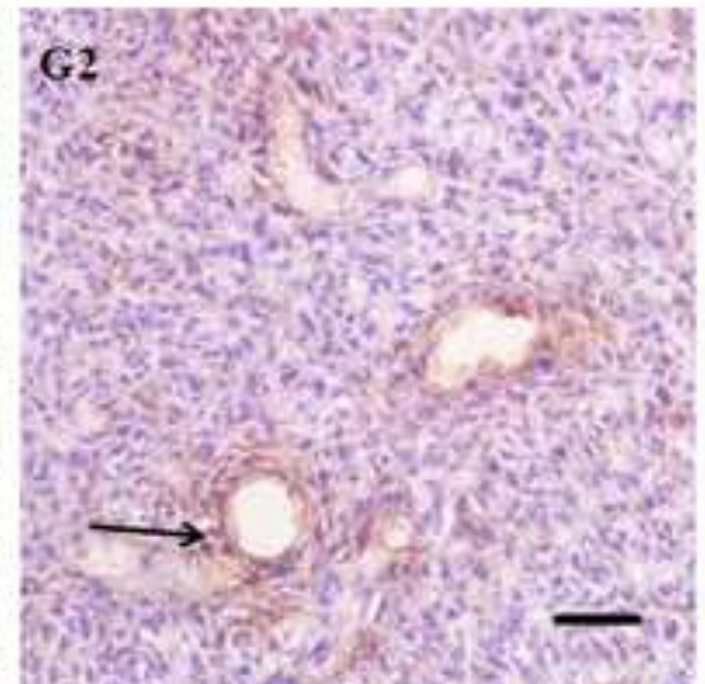

G3

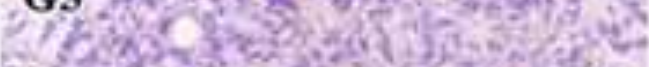

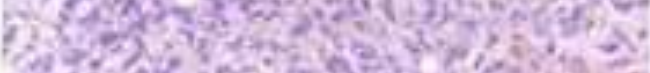

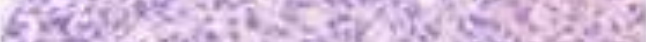

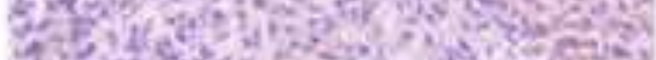

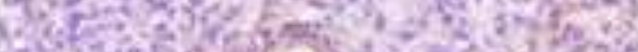

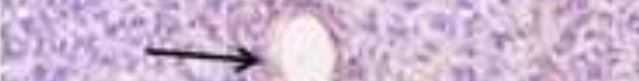

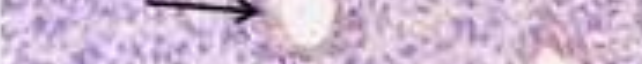

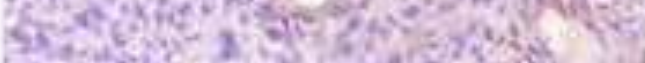

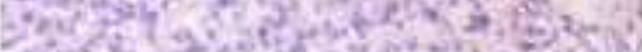

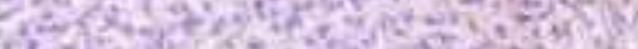

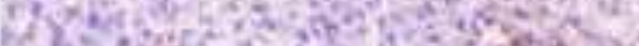

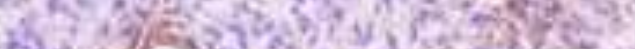

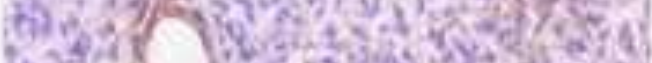

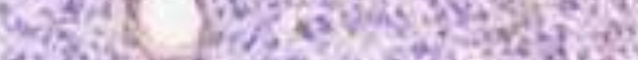

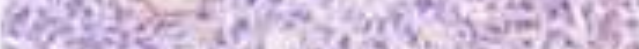
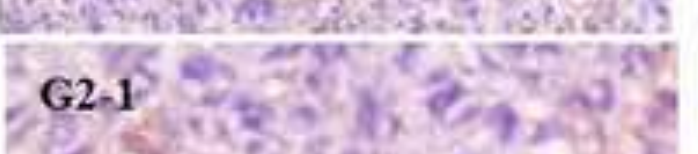

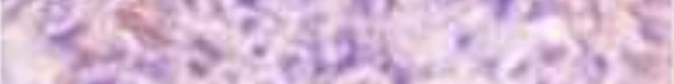

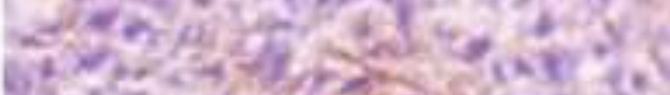

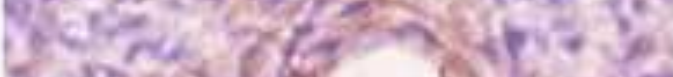

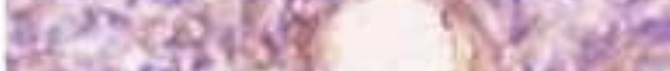

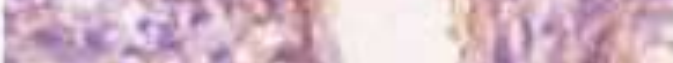
7.

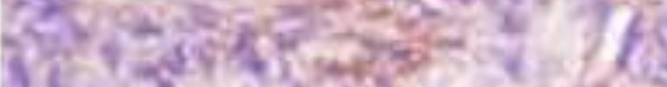

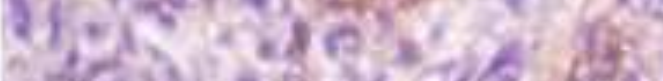

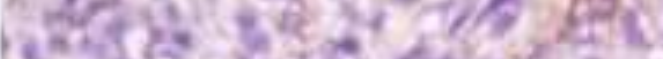

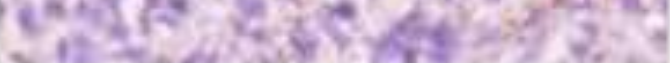

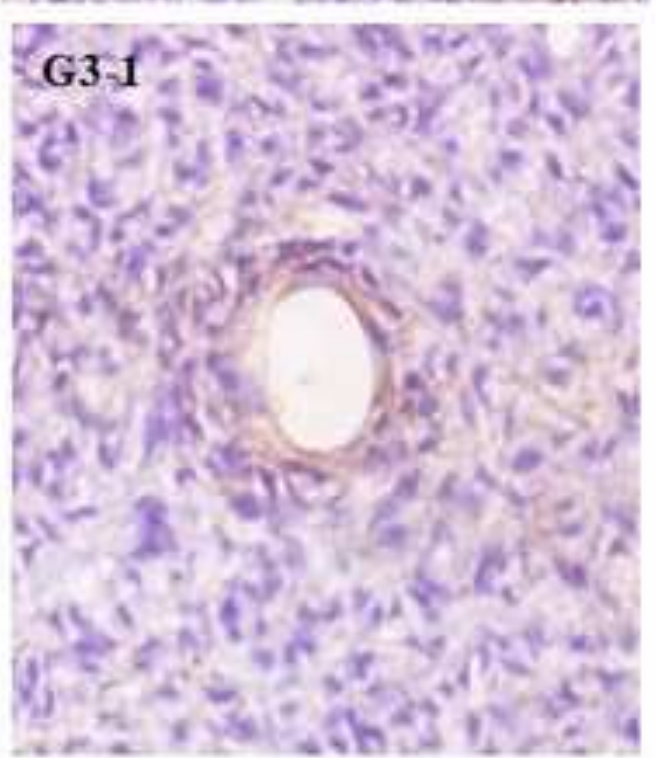


Figure $2 \mathrm{H}$

H

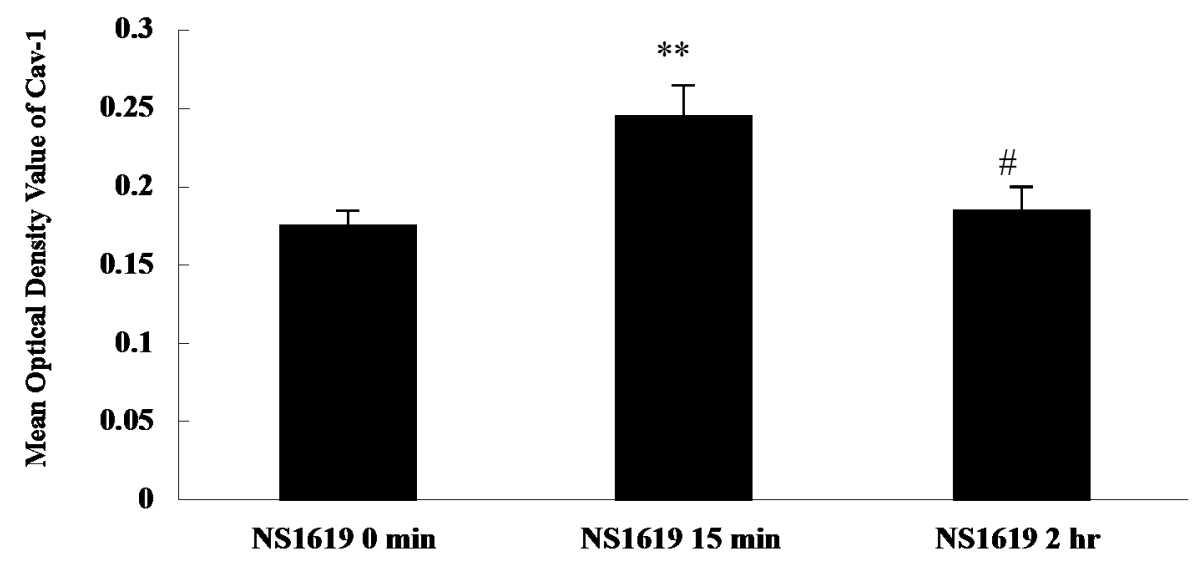

NS1619 0 min

NS1619 15 min

NS1619 2 hr 
Figure 3

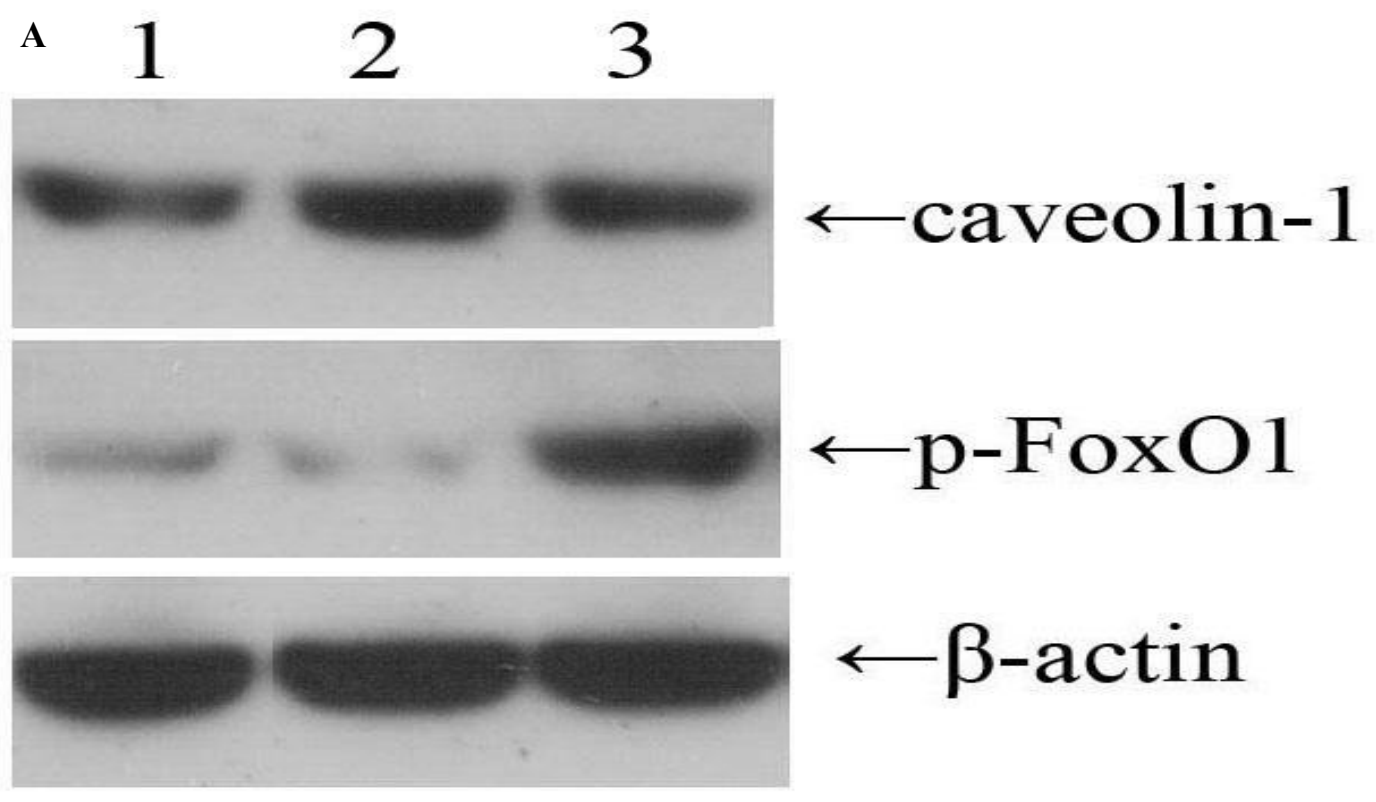

B
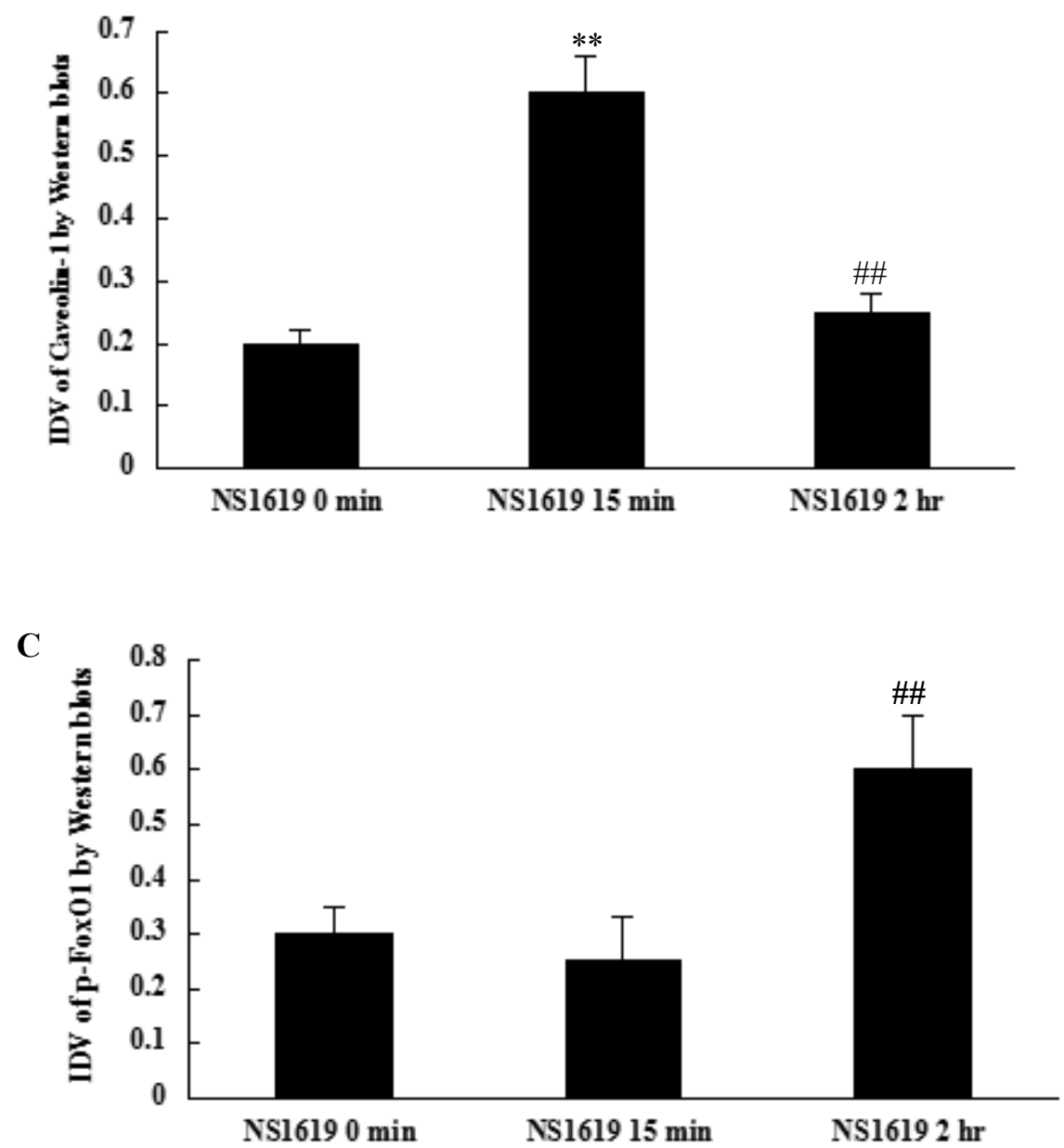

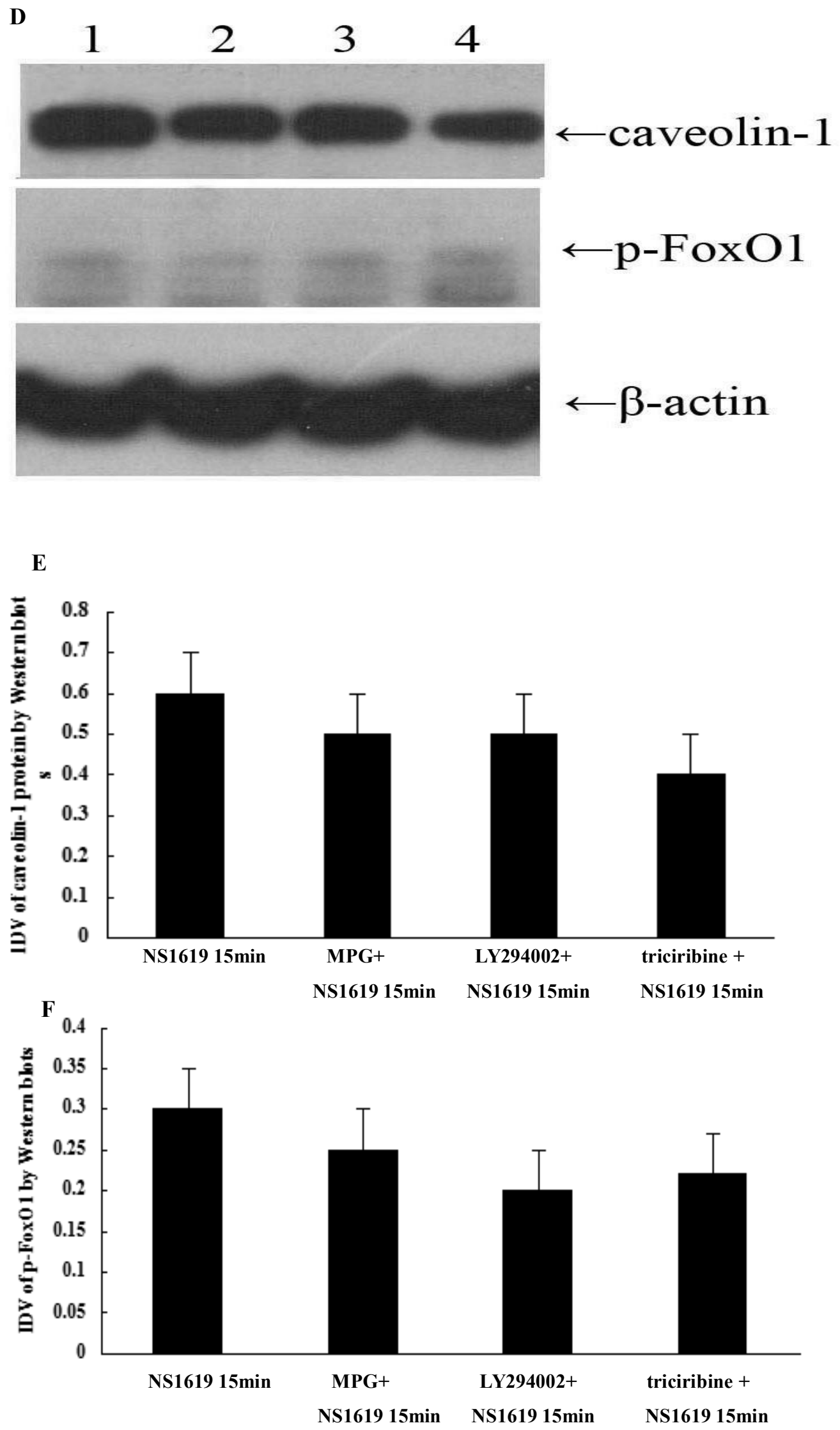

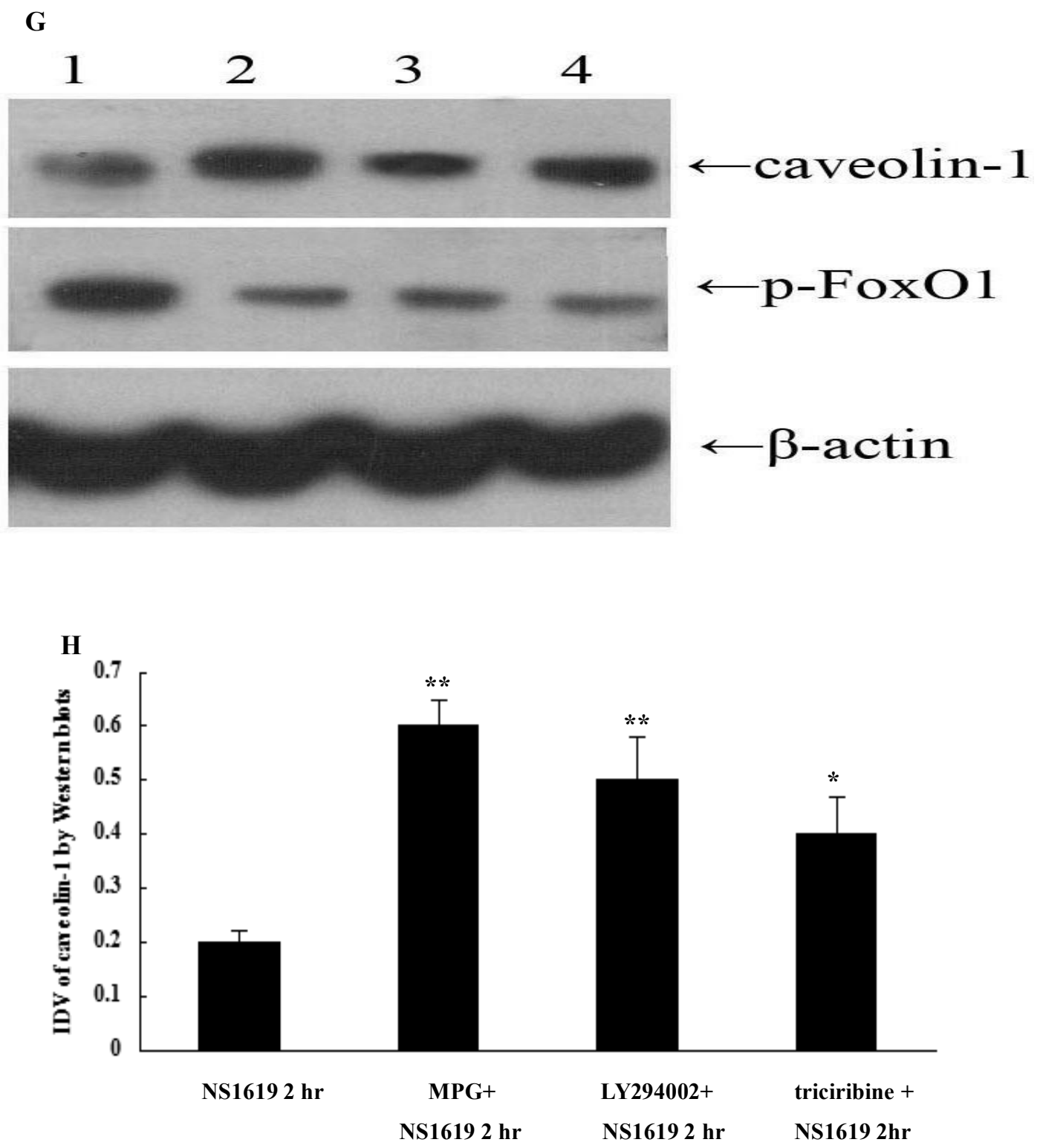

I

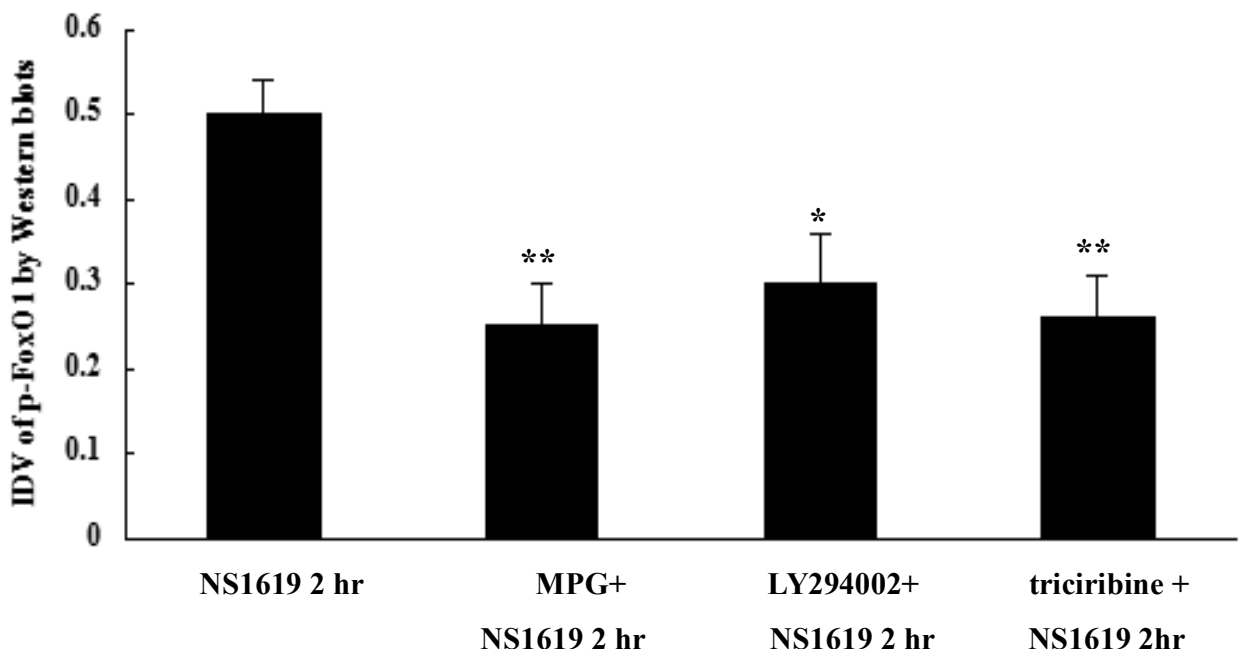


Caveolin-1
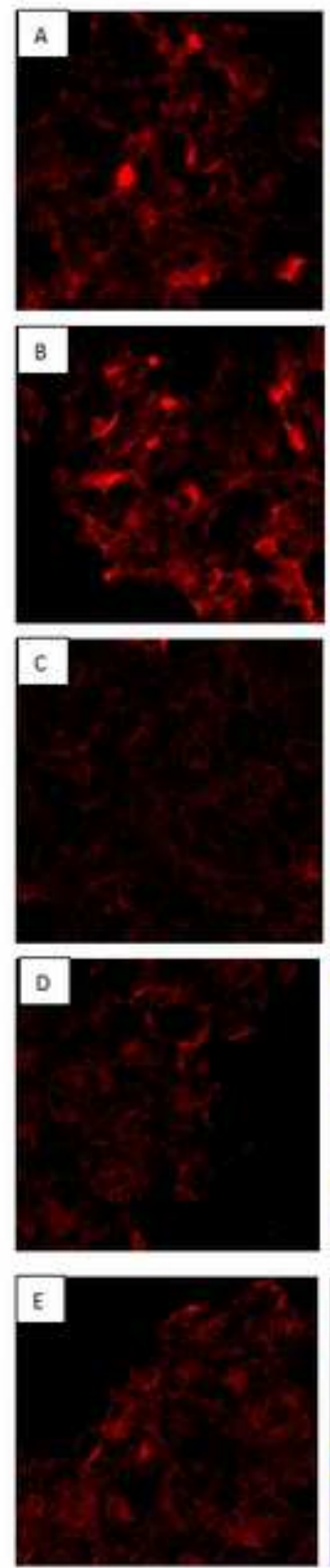

DAPI
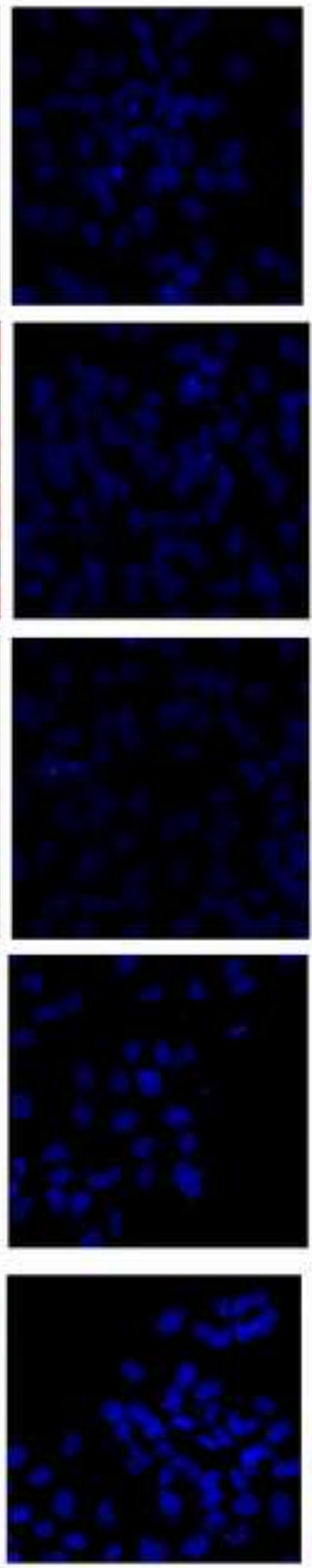

Merge
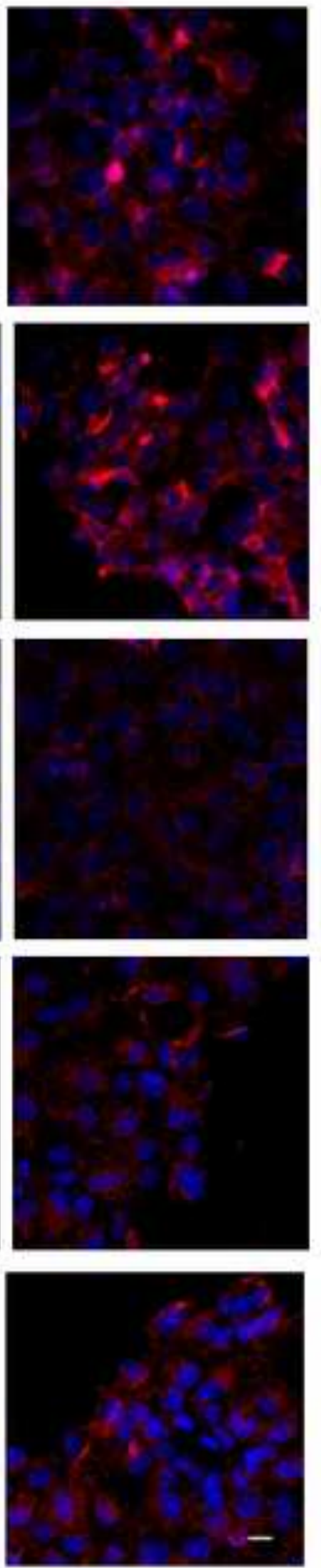